Published in final edited form as:

Aging Cell. 2010 August ; 9(4): 457-461. doi:10.1111/j.1474-9726.2010.00592.x.

\title{
Hot topics in stem cells and self-renewal: 2010
}

\author{
Norman E. Sharpless \\ Departments of Medicine and Genetics, The University of North Carolina School of Medicine, The \\ Lineberger Comprehensive Cancer Center, The University of North Carolina, CB\# 7295, Chapel \\ Hill, NC 27599-7295, USA
}

\section{Summary}

In many tissues, mammalian aging is associated with a decline in the replicative and functional capacity of somatic stem cells and other self-renewing compartments. Understanding the basis of this decline is a major goal of aging research. In particular, therapeutic approaches to ameliorate or reverse the age-associated loss of stem function could be of use in clinical geriatrics. Such approaches include attempts to protect stem cells from age-promoting damage, to 'rejuvenate' stem cells through the use of pharmacologic agents that mitigate aging-induced alterations in signaling, and to replace lost stem cells through regenerative medicine approaches. Some headway has been made in each of these arenas over the last 18 months including advances in the production of donor-specific totipotent stem cells through induced pluripotency (iPS), gains in our understanding of how tumor suppressor signaling is controlled in self-renewing compartments to regulate aging, and further demonstration of extracellular 'milieu' factors that perturb stem cell function with age. This period has also been marked by the recent award of the Nobel Prize in Physiology or Medicine for elucidation of telomeres and telomerase, a topic of critical importance to stem cell aging.

\section{Keywords}

aging; induced pluripotency; senescence; telomere

There have been several recent reports in stem cell and self-renewal biology of relevance to aging. Although any summary of such a wide topic as stem cell biology necessarily omits many important advances, I will summarize a few of the more notable findings over the last 18 months. The major findings in this area with regard to aging can be broadly grouped into two general areas:

1. Insights into the mechanism of reprogramming and induced pluripotency (iPS)

2. Insights into the aging of somatic stem cells.

\section{Regenerative medicine and iPS}

Induced pluripotency refers to the stable reprogramming of somatic cells into a state that is similar to that of embryonic stem (ES) cells. Induced pluripotency cells have many of the properties of ES cells, including the ability to differentiate into several somatic tissues. Methods to make such donor-specific totipotent cells are expected to be of high importance for the future of regenerative medicine (Kiskinis \& Eggan, 2010). The initial procedure for

\footnotetext{
(C) 2010 The Author

Correspondence Norman E. Sharpless, Departments of Medicine and Genetics, The University of North Carolina School of Medicine, The Lineberger Comprehensive Cancer Center, The University of North Carolina, CB\# 7295, Chapel Hill, NC 27599-7295, USA. Tel.: 919966 1185; fax: 919966 8212; nes@ med.unc.edu.
} 
inducing reprogramming involved retroviral transduction with 3-5 genes (Oct4, Sox2, Myc, Klf4, Lin28), but this method is not ideal for human use, because it is inefficient and potentially oncogenic (e.g., requiring retroviral insertion, especially with potentially oncogenic genes such as c-Myc). Several recent papers have enhanced our understanding of reprogramming, showing that cell cycle inhibitors decrease the efficiency of reprogramming of human and murine somatic cells.

The initial finding in this regard came out of a small candidate RNAi screen for genes whose knockdown facilitated reprogramming (Zhao et al., 2008). In this work, the authors found that knockdown of p53 significantly enhanced the reprogramming of murine somatic cells. This provocative observation was followed by a spate of papers employing a variety of approaches in human and murine systems that collectively demonstrated that activation of two archetypal tumor suppressor pathways, ARF-p53 and p16 ${ }^{\mathrm{INK} 4 \mathrm{a}}-\mathrm{RB}$, decreases reprogramming of somatic cells (Hong et al., 2009; Kawamura et al., 2009; Li et al., 2009; Marion et al., 2009; Utikal et al., 2009). The act of reprogramming appears to induce expression of the INK4al ARF locus that encodes p16 $6^{\mathrm{INK} 4 \mathrm{a}}$ and ARF. Both proteins decrease cell cycle traversal by inhibiting cyclin-dependent kinase activity, the latter by activating p53 and its downstream effector, p21 CIP. Inactivation of these anti-proliferative mechanisms through a variety of means enhanced reprogramming efficiency. Loss of p53 increased the efficiency of reprogramming and obviated the need for Klf4 and Myc, permitting reprogramming with Oct4 and Sox 2 only, and suggesting that part of the function of Myc and KLF4 in reprogramming may be to increase cell cycle traversal. Inhibition of p53 enhanced reprogramming of both human and murine cells, but a species difference was observed with regard to products of the INK4al $A R F$ locus: ARF appeared to be a more important barrier to reprogramming in murine cells, whereas $\mathrm{p} 16^{\mathrm{INK} 4 \mathrm{a}}$ was more important in human cell reprogramming. Permanent inactivation of these tumor suppressor mechanisms would clearly be undesirable for cells used in human therapeutics, but work from these groups suggested that persistent inactivation of p53 and the INK4al $A R F$ locus was not required once reprogramming was established. A future question suggested by these papers is whether transient pharmacologic inhibition of $\mathrm{p} 53$ and/or $\mathrm{p} 16^{\mathrm{INK} 4 \mathrm{a}}$ can also facilitate reprogramming.

Of particular importance to gerontology, reprogramming efficiency was decreased using skin fibroblasts from old compared to young mice. Prior work had shown such cells markedly upregulate the expression of Ink4al Arf locus with aging, and Serrano and colleagues gratifyingly showed that shRNA targeting of the locus rescued the reprogramming defect in elderly cells ( $\mathrm{Li}$ et al., 2009). Similarly, telomere dysfunction and other forms of DNA damage, which may be more prevalent in cells from elderly individuals, were also shown to decrease the efficiency of reprogramming in a p53-dependent manner (Marion et al., 2009). Subsequent work from the Jaenisch laboratory further refined these observations, demonstrating that iPS reprogramming requires the acquisition of stochastic epigenetic events, some of which are independent and others dependent on cell cycle traversal (Hanna et al., 2009). Therefore, events such as loss of p53 may enhance reprogramming by increasing proliferation, whereas expression of genes like Nanog appears to enhance reprogramming independently of an effect on the cell cycle.

\section{Somatic self-renewal and aging Hematopoietic stem and progenitor cell}

A long-standing debate in the stem cell aging field has focused on which changes in stem cell function are 'intrinsic' vs. 'extrinsic'. Intrinsic changes are those localized within the stem cell and its progeny, e.g., telomere shortening or some heritable changes in epigenetic state. The complex exchange of information from the interior and exterior of a cell may blur 
this distinction, but the common assumption among stem cell experts has held for some time that major changes in stem cell function with aging are intrinsic. In 2005, this notion was questioned by Conboy, Wagers, Rando and colleagues, who used elegant parabiosis experiments to demonstrate that muscle stem cell aging was largely governed by milieu (Conboy et al., 2005). Their approach was to conjoin the blood supply of old and young mice to evaluate the effects of the old milieu on the young mouse (and vice versa).

One of the strongest bits of evidence for intrinsic aging has been the often-reproduced finding that old hematopoietic stem cells (HSC) transplanted into young mice retain characteristics of old cells; that is, HSC are not reprogrammed by the young niche into younger stem cells [reviewed in (Rossi \& Sharpless, 2008)]. A criticism of this approach has been that transplantation requires 'conditioning' of the recipient (e.g., through whole-body irradiation) and therefore necessarily entails damage to the niche. The question of HSC aging has now been further pursued by the Wagers laboratory using parabiosis, which does not require conditioning (Mayack et al., 2010). As is the case with muscle stem cell function, the authors showed that a considerable component of HSC aging results from an aged stem cell niche. Many of the properties of old HSC were rescued by young serum and/ or a young microenvironment. The reconciliation of these results with the transplantation results, as well as with studies by Rossi, Weissman and colleagues showing increased DNA damage in normal HSC with aging (Rossi et al., 2007), is still not presently clear but is an area of active ongoing study.

An important feature of the aged HSC is the so-called myeloid skewing, an increase in the ability of old bone marrow to produce myeloid cell types (e.g., granulocytes) with a decreased ability to produce cells of the lymphocytic lineage [reviewed in (Rossi \& Sharpless, 2008)]. The conventional explanation for this observation has been that all HSC experience a change in lineage preference from lymphoid to myeloid elements with aging. Rossi and colleagues have recently suggested an alternative model to explain this finding (Beerman et al., 2010). They demonstrated the existence of distinct subtypes of HSC that differ in lineage preference and which can be distinguished based on cell surface phenotype. Importantly, these HSC subtypes appear to differ in their ability to generate myeloid vs. lymphoid progeny. With regard to aging, the authors observed an expansion of the myeloidtype HSC with aging, possibly at the expense of lymphoid- biased cells. This new model suggests that aging may not alter the lineage preference of individuals HSC but instead may change the proportions of HSC with a myeloid vs. lymphoid bias.

\section{Pancreatic $\beta$-cells}

Expression of the INK4al $A R F$ locus inhibits self-renewal in some stem cells and other selfrenewing compartments with aging, but the factors responsible for the age-related induction of INK4al ARF locus are not known. Two recent papers addressed this question in one of the best defined systems to study the effects of INK4al $A R F$ expression on proliferation with aging, the pancreatic $\beta$-cell. The Kim and Bhushan laboratories independently showed that repression of the INK4al $A R F$ locus by members of the Polycomb group (PcG) family such as BMI1 and EZH2 is required to repress INK4al ARF in vivo (Chen et al., 2009; Dhawan et al., 2009). Loss of PcG function was associated with $\beta$-cell hypoplasia and diabetes and could be rescued by concomitant Ink4al Arf deficiency. Importantly, a loss of EZH2 expression in human $\beta$-cells with aging was also observed, and this loss of expression was inversely correlated with increased $\mathrm{p} 16^{\mathrm{INK} 4 \mathrm{a}}$ expression in the same tissues. Therefore, these papers suggest that loss of PcG repression, a well-recognized event in developmental biology also plays an important role in regulating the proliferative capacity of an important self-renewing tissue with age. 
A related finding suggests a possible rationale for the ineffectiveness, in older patients, of a new class of antidiabetic agents. There has been controversy among diabetologists as to the role of decreased $\beta$-cell replication with aging in type 2 diabetes (T2DM) in humans, but several lines of evidence, including recent genome-wide association studies, have suggested that impaired $\beta$-cell replication is a major contributor to T2DM onset. Glucagon-Like Peptide-1 (GLP1) agonists, a new class of therapeutic for T2DM, seem to be able both to increase peripheral insulin sensitivity and also to augment $\beta$-cell proliferation. Rankin and Kushner have now shown that the ability to regenerate islets is severely compromised by aging in mice, finding that GLP1 agonists potently induced $\beta$-cell replication in young, but not old, mice (Rankin \& Kushner, 2009). GLP-1 agonists thus represent a clear example of how therapeutics that enhance self-renewal can be effective in a common human disease but at the same time show how age-associated decline in replicative function might cause such agents to lose efficacy in older individuals.

\section{Other somatic stem and progenitor cell aging}

One of the oldest and mot contentious problems in somatic stem cell biology has been the delineation of which adult mammalian tissues possess regenerative capacity, presumably reflecting resident stem cell function. Strong assertions as to the absence or presence of tissue-specific stem cells have been made in many tissues, often based on incomplete experimental data. 'Lineage tracing' approaches have been used with some success in animal models to show cellular regeneration exists where none was expected, and vice versa. Such an approach, however, has not been available for the study of humans. A recent study by Frisen and colleagues presents a new method to study myocardial turnover in the adult human heart (Bergmann et al., 2009). By testing cardiac DNA for content of carbon-14 generated during the Second World War, the authors were able to estimate myocardial turnover in humans. The results suggested a $1 \%$ turnover rate per year in young adults that falls to $<0.5 \%$ in older adults, implying turnover of $<50 \%$ of myocardial cells during a human lifespan. While this amount is far less than claimed by some groups, it provides clear evidence for some modest capacity for myocardial replacement in adults and suggests the possibility that this limited regenerative capacity could be therapeutically enhanced.

Skeletal muscle has well-defined stem/progenitor cells that are thought to self-renew throughout the normal life span. Prior work has shown that the age-associated loss of function of muscle progenitors is largely determined by external cues and aged muscle progenitors can be rescued by transplantation into a young microenvironment (Conboy et al., 2005). Given these findings, there has been an ongoing search for hormonal and niche factors that cause muscle progenitor dysfunction in old mice, or alternatively factors that enhance the function of these cells in young animals. This effort took an interesting turn when the Conboy laboratory showed that TGF- $\beta$ signaling induces the expression of cell cycle inhibitors such as $\mathrm{p} 15^{\mathrm{INK} 4 \mathrm{~b}}, \mathrm{p} 16^{\mathrm{INK} 4 \mathrm{a}}$, and $\mathrm{p} 27^{\mathrm{KIP}}$, a plausible mechanism to explain the cell cycle defect of aged muscle progenitors (Carlson et al., 2008). Importantly, in this and subsequent work (Carlson et al., 2009), the group showed that inhibitors of TGF- $\beta$ signaling could enhance muscle progenitor function in standard in vivo muscle regeneration assays. These results are promising, given the importance of sarcopenia in aging populations and the recent attention of pharmaceutical firms to development of candidate small molecule inhibitors of TGF- $\beta$.

Dorshkind, Witte and colleagues suggested an intriguing link between cancer and aging in a study of early B-lymphocyte progenitors (Signer et al., 2008). They observed a marked increase in Ink4al Arf expression in these cells with aging and found that expression of this tumor suppressor locus correlated with a decreased transformability of these cells with aging. While at first such a result seems counterintuitive (in general, the incidence of cancer increases with aging), the authors correctly point out that some forms of B-cell leukemia are 
pediatric diseases that decrease in frequency with aging. Their work implies a trade-off between the ability to generate new B cells and the ability to produce a B-cell neoplasm and suggest this dichotomy is regulated by the activity of classic tumor suppressor mechanisms such as expression of $\mathrm{p} 16^{\mathrm{INK} 4 \mathrm{a}}$ and ARF.

It has been thought that a decrease in melanocyte self-renewal occurs with aging and plays an important role in graying, a familiar age-associated phenotype. Expression of p16 ${ }^{\mathrm{INK} 4 \mathrm{a}}$, which promotes cellular senescence, is an indisputable barrier to melanocyte transformation, and other groups have suggested an increase in melanocyte senescence might play some role in graying. Nishimura and colleagues have recently challenged this model, however, suggesting that genotoxic stress reduces melanocyte self-renewal by inducing aberrant differentiation of melanocytic stem cells (MSC) (Inomata et al., 2009). These aberrantly differentiated MSC remain within the normal MSC niche but are functionally deficient in the production of new melanocytes. This process was not influenced by genetic inactivation of classical effectors of senescence or apoptosis, such as Ink4al Arf expression, but was accelerated by loss of ataxia-telangiectasia mutated, an important sensor of DNA damage. This work suggests an intrinsic replicative failure of a stem cell compartment with aging that does not result from senescence or apoptosis but instead results from a newly described form of hypoproliferation associated with aberrant differentiation.

With regard to the role of telomere dysfunction in human aging, the story of patients with congenital telomerase deficiency continues to evolve. Adding to data that germline telomere insufficiency is associated with dyskeratosis congenita, aplastic anemia (AA), idiopathic pulmonary fibrosis (IPF), and predilection to certain cancers, Lansdorp, Young and colleagues have recently reported an increased incidence of fibrotic liver disease in individuals with telomerase mutations (Calado et al., 2009). These findings are in line with prior studies in the mouse (Rudolph et al., 2000) and may explain the link between states of chronic liver turnover (e.g., hepatitis) and cirrhosis. Bone marrow hypoplasia and pulmonary fibrosis do increase in frequency with normal aging, but symptomatic disease from either condition is relatively rare. Fibrotic liver disease such as cirrhosis, however, is relatively common in the general population. These results thus open the possibility that telomere biology may be a general contributor to physiologic human hepatic aging.

Researchers with an interest in somatic stem cell biology were particularly pleased to note the recent awarding of the 2009 Nobel Prize in Physiology or Medicine to Blackburn, Greider and Szostak for their pioneering work in understanding telomeres and telomerase. The work of these pioneers has had a profound impact on our understanding of human diseases such as AA and IPF, as well as contributed to a more general understanding of cancer and aging.

\section{Future questions}

What discoveries will headline the Hot Topics review on aging and stem cell biology in 2013? Predicting even the very near future is fraught with peril in such a fast moving area, but I believe a few topics in stem cell biology will see considerable progress over the next 24 months. The significant technical hurdles associated with iPS reprogramming, which limit the clinical use of this technology, certainly need to be addressed. In particular, a mechanism to reprogram cells that does not rely on the use of retroviral transduction or stable integration seems an achievable goal, with success already reported using plasmid vectors (Okita et al., 2010) or RNA transfection (Yakubov et al., 2010). Likewise, if integration-free mechanisms to induce iPS cells to differentiate into desired tissues can be developed and refined, and such approaches can be validated and scaled for clinical use, one expects the therapeutic testing of patient-specific, iPS-derived cell-based therapies in the 
near term, although the pace of clinical trials is likely to disappoint those used to the thrills of basic science.

Cell-based therapeutics that rely on iPS cells, ES cells, or transplantation of somatic stem cells may be expensive and still confront technical challenges that may turn out to be considerable impediments to human use. Even were these issues all resolved in short order, the potential toxicity (e.g., long-term cancer risk) could make such approaches a last resort for most patients. Therefore, there continues to be considerable enthusiasm about mechanisms to protect and extend the longevity of tissue-specific regenerative cells, similar to the aforementioned work with GLP1 agonists in $\beta$-cells and TGF- $\beta$ inhibitors in muscle. Equally important will be an understanding of the toxicity of such approaches, especially as such methods are envisioned for long-term use. Several groups are also examining the beneficial effect of more generally conceived 'anti-aging' therapies on self-renewing compartments. For example, do the apparent anti-aging benefits of rapamycin reflect enhanced stem cell function in aged mice?

It is often difficult to discover which age-related phenotypes result from stem cell dysfunction, reflecting the more general difficulty of determining which organs do indeed have significant regenerative capacity. The adult heart, brain, and islet were all tissues formerly thought to have little significant regenerative capacity in adult humans, but this notion has now been largely debunked for each of these tissues. Even very small amounts of daily proliferation can be significant over a mammalian life span for preservation of tissue homeostasis. In addition, many regenerative tissue compartments exhibit a 'facultative' behavior, behaving as stem cells under certain conditions of stress, but otherwise remaining largely quiescent. Challenges in identifying tissues with adult stem and progenitor cells complicate the analysis of what aspects of aging result from dysfunction or failure of resident self-renewing cells. I believe that over the next few years ongoing work in several models of age-related pathology will document the involvement of age-related stem cell dysfunction and suggest new approaches to manipulate stem cell biology to the benefit of patients vulnerable to age-related illnesses.

\section{Acknowledgments}

NES is supported by grants from the NIA (AG024379) and a Burroughs Wellcome Fund Clinical Scientist in Translational Research Award.

\section{References}

Beerman I, Bhattacharya D, Zandi S, Sigvardsson M, Weissman IL, Bryder D, Rossi DJ. Functionally distinct hematopoietic stem cells modulate hematopoietic lineage potential during aging by a mechanism of clonal expansion. Proc Natl Acad Sci USA. 2010; 107:5465-5470. [PubMed: 20304793]

Bergmann O, Bhardwaj RD, Bernard S, Zdunek S, Barnabe-Heider F, Walsh S, Zupicich J, Alkass K, Buchholz BA, Druid H, Jovinge S, Frisen J. Evidence for cardiomyocyte renewal in humans. Science (New York, NY). 2009; 324:98-102.

Calado RT, Regal JA, Kleiner DE, Schrump DS, Peterson NR, Pons V, Chanock SJ, Lansdorp PM, Young NS. A spectrum of severe familial liver disorders associate with telomerase mutations. PLoS ONE. 2009; 4:e7926. [PubMed: 19936245]

Carlson ME, Hsu M, Conboy IM. Imbalance between pSmad3 and Notch induces CDK inhibitors in old muscle stem cells. Nature. 2008; 454:528-532. [PubMed: 18552838]

Carlson ME, Conboy MJ, Hsu M, Barchas L, Jeong J, Agrawal A, Mikels AJ, Agrawal S, Schaffer DV, Conboy IM. Relative roles of TGF-beta1 and Wnt in the systemic regulation and aging of satellite cell responses. Aging cell. 2009; 8:676-689. [PubMed: 19732043] 
Chen H, Gu X, Su IH, Bottino R, Contreras JL, Tarakhovsky A, Kim SK. Polycomb protein Ezh2 regulates pancreatic beta-cell Ink4a/Arf expression and regeneration in diabetes mellitus. Genes Dev. 2009; 23:975-985. [PubMed: 19390090]

Conboy IM, Conboy MJ, Wagers AJ, Girma ER, Weissman IL, Rando TA. Rejuvenation of aged progenitor cells by exposure to a young systemic environment. Nature. 2005; 433:760-764. [PubMed: 15716955]

Dhawan S, Tschen SI, Bhushan A. Bmi-1 regulates the Ink4a/Arf locus to control pancreatic beta-cell proliferation. Genes Dev. 2009; 23:906-911. [PubMed: 19390085]

Hanna J, Saha K, Pando B, van Zon J, Lengner CJ, Creyghton MP, van Oudenaarden A, Jaenisch R. Direct cell reprogramming is a stochastic process amenable to acceleration. Nature. 2009; 462:595601. [PubMed: 19898493]

Hong H, Takahashi K, Ichisaka T, Aoi T, Kanagawa O, Nakagawa M, Okita K, Yamanaka S. Suppression of induced pluripotent stem cell generation by the p53-p21 pathway. Nature. 2009; 460:1132-1135. [PubMed: 19668191]

Inomata K, Aoto T, Binh NT, Okamoto N, Tanimura S, Wakayama T, Iseki S, Hara E, Masunaga T, Shimizu H, Nishimura EK. Genotoxic stress abrogates renewal of melanocyte stem cells by triggering their differentiation. Cell. 2009; 137:1088-1099. [PubMed: 19524511]

Kawamura T, Suzuki J, Wang YV, Menendez S, Morera LB, Raya A, Wahl GM, Belmonte JC. Linking the p53 tumour suppressor pathway to somatic cell reprogramming. Nature. 2009; 460:1140-1144. [PubMed: 19668186]

Kiskinis E, Eggan K. Progress toward the clinical application of patient-specific pluripotent stem cells. J Clin Invest. 2010; 120:51-59. [PubMed: 20051636]

Li H, Collado M, Villasante A, Strati K, Ortega S, Canamero M, Blasco MA, Serrano M. The Ink4/Arf locus is a barrier for iPS cell reprogramming. Nature. 2009; 460:1136-1139. [PubMed: 19668188]

Marion RM, Strati K, Li H, Murga M, Blanco R, Ortega S, Fernandez-Capetillo O, Serrano M, Blasco MA. A p53-mediated DNA damage response limits reprogramming to ensure iPS cell genomic integrity. Nature. 2009; 460:1149-1153. [PubMed: 19668189]

Mayack SR, Shadrach JL, Kim FS, Wagers AJ. Systemic signals regulate ageing and rejuvenation of blood stem cell niches. Nature. 2010; 463:495-500. [PubMed: 20110993]

Okita K, Hong H, Takahashi K, Yamanaka S. Generation of mouse-induced pluripotent stem cells with plasmid vectors. Nat Protoc. 2010; 5:418-428. [PubMed: 20203661]

Rankin MM, Kushner JA. Adaptive beta-cell proliferation is severely restricted with advanced age. Diabetes. 2009; 58:1365-1372. [PubMed: 19265026]

Rossi, DJ.; Sharpless, NE. Aging in mammalian stem cells and other self-renewing compartments. In: Guarente, L.; Partridge, L., editors. The Molecular Biology of Aging. Cold Spring Harbor, NY: Cold Spring Harbor Press; 2008. p. 237-267.

Rossi DJ, Bryder D, Seita J, Nussenzweig A, Hoeijmakers J, Weissman IL. Deficiencies in DNA damage repair limit the function of haematopoietic stem cells with age. Nature. 2007; 447:725729. [PubMed: 17554309]

Rudolph KL, Chang S, Millard M, Schreiber-Agus N, DePinho RA. Inhibition of experimental liver cirrhosis in mice by telomerase gene delivery. Science. 2000; 287:1253-1258. [PubMed: 10678830]

Signer RA, Montecino-Rodriguez E, Witte ON, Dorshkind K. Aging and cancer resistance in lymphoid progenitors are linked processes conferred by p16Ink4a and Arf. Genes Dev. 2008; 22:3115-3120. [PubMed: 19056891]

Utikal J, Polo JM, Stadtfeld M, Maherali N, Kulalert W, Walsh RM, Khalil A, Rheinwald JG, Hochedlinger K. Immortalization eliminates a roadblock during cellular reprogramming into iPS cells. Nature. 2009; 460:1145-1148. [PubMed: 19668190]

Yakubov E, Rechavi G, Rozenblatt S, Givol D. Reprogramming of human fibroblasts to pluripotent stem cells using mRNA of four transcription factors. Biochem Biophys Res Commun. 2010; 394:189-193. [PubMed: 20188704]

Zhao Y, Yin X, Qin H, Zhu F, Liu H, Yang W, Zhang Q, Xiang C, Hou P, Song Z, Liu Y, Yong J, Zhang P, Cai J, Liu M, Li H, Li Y, Qu X, Cui K, Zhang W, Xiang T, Wu Y, Zhao Y, Liu C, Yu C, 
Yunn K, Lou J, Ding M, Deng H. Two supporting factors greatly improve the efficiency of human iPSC generation. Cell Stem Cell. 2008; 3:475-479. [PubMed: 18983962] 